This item was submitted to Loughborough's Research Repository by the author.

Items in Figshare are protected by copyright, with all rights reserved, unless otherwise indicated.

\title{
The low current electrochemical mechanisms of the fuel cell cathode catalyst layer through an impedance study
}

\section{PLEASE CITE THE PUBLISHED VERSION}

http://dx.doi.org/10.1149/1.3280267

\section{PUBLISHER}

(c) Electrochemical Society

\section{VERSION}

AM (Accepted Manuscript)

\section{PUBLISHER STATEMENT}

This work is made available according to the conditions of the Creative Commons Attribution-NonCommercialNoDerivatives 4.0 International (CC BY-NC-ND 4.0) licence. Full details of this licence are available at: https://creativecommons.org/licenses/by-nc-nd/4.0/

\section{LICENCE}

CC BY-NC-ND 4.0

\section{REPOSITORY RECORD}

Cruz-Manzo, Samuel, Pratap Rama, and Rui Chen. 2015. "The Low Current Electrochemical Mechanisms of the Fuel Cell Cathode Catalyst Layer Through an Impedance Study". figshare. https://hdl.handle.net/2134/18548. 


\title{
The Low Current Electrochemical Mechanisms of the Fuel Cell Cathode Catalyst Layer through an Impedance Study
}

\author{
Samuel Cruz-Manzo, Pratap Rama, Rui Chen \\ Department of Aeronautical and Automotive Engineering, Loughborough University, UK
}

\begin{abstract}
Based on the fundamental electrode theory and the impedance experimental study, a numerical model to simulate the low current distribution in the time domain and the electrochemical impedance spectra of the Cathode Catalyst Layer (CCL) of Polymer Electrolyte Fuel Cells (PEFCs) has been developed in this study. The model development consists of two stages, to establish the fundamental equations for the low current distribution in the $\mathrm{CCL}$ in the time domain, and to resolve the fundamental theory in the frequency domain. It was validated by comparing the simulated impedance of the CCL directly against the impedance data measured from an operational test cell. The simulated frequency response agrees well with the experimental data. The model was applied in the time domain to simulate the effects of proton resistance and the double layer capacitance across the CCL on the transitory and steady-state current distribution. The results showed that the model has established a backbone understanding of how the low current electrochemical mechanisms relate to the electrochemical impedance spectra of the CCL. It establishes a wider scope to relate the electrochemical impedance data to the fundamental theory of PEFCs.
\end{abstract}

Keywords: Fuel Cell - PEM, Cathode Catalyst Layer, Electrochemical Impedance Spectroscopy 


\section{Introduction}

The Polymer Electrolyte Fuel Cell (PEFC) is an electrochemical energy conversion device which converts the chemical energy that is released during an oxygen-reduction and hydrogen-oxidation (redox) process into usable electrical energy and thermal energy. The redox process occurs electrochemically in the catalyst-based electrodes of the cell and forgoes any form of combustion; the PEFC is as such theoretically unbound by the Carnot limit. In general, the catalyst layer is formed by a network of carbon particles that provide pathways for electron conduction. In addition, the catalyst layer contains a dispersion of polymer electrolyte to ensure continuity for proton conduction via the PEM. The resulting matrix structure is porous in nature and characterised by tortuous pathways for reactant transport.

Electrochemical reactions in the fuel cell rely upon the utilisation of $\mathrm{Pt}$ in the catalyst layer. The literature suggests that it is possible to achieve a platinum utilisation of $86-87 \%$ [1]. One of the most important challenges is to obtain the highest electrochemically surface area with the minimum amount of the catalyst. It has been proven that a simultaneous increase of electrochemical activity and rate of species transport cannot be obtained with a single homogeneous phase [2]. Furthermore, improving one property of the catalyst layer can adversely affect another. For instance, by increasing the amount of the electrolyte between agglomerates the proton conductivity can be enhanced, but will also close void spaces for reactant transport. Developing catalyst layers with smaller agglomerated structures can increase the electrode- electrolyte interface but can compromise secondary pores. An optimal catalyst layer structure is thus difficult to achieve.

To enhance the understanding of the factors governing the performance of catalyst layer structures and therefore how to engineer optimal catalyst layer structures, it is necessary to harness capabilities from both modelling and experimental techniques in a complementary manner. Electrochemical Impedance Spectroscopy (EIS) is a non-invasive experimental technique which measures the opposition to the flow of an Alternating Current (AC) within a system which contains elements that dissipate energy and store energy. The opposition to the AC load, or impedance, can be measured over a range of frequencies, which thereby reveals the frequency response of the system. When subjecting a PEFC to a sinusoidal input (voltage), a sinusoidal output (current) is generated at the same frequency as the input but with a different amplitude and phase. The PEFC can be characterised in the frequency domain by measuring both the amplitude ratio of the input sinusoid (voltage) to the output sinusoid (current) and the phase shift as functions of the frequency. The energy dissipating and energy storing elements of the cell that result in the observed frequency response can be represented by an equivalent circuit model that is composed of resistors and capacitors respectively. The magnitude of these components are tuned in order to fit the simulated frequency response of that circuit to the measured frequency response of the PEFC. By understanding the arrangement and magnitude of the resistive and capacitive elements in the equivalent circuit, it is possible to generate a deeper understanding of how and where the electrical energy that is generated due to the redox reaction is being dissipated and retained within the real physical system.

The literature demonstrates that the EIS technique has been widely applied to characterise and evaluate electrochemical systems, particularly PEFCs. Romero et al. [3] applied this approach to demonstrate that the main 
characteristics in fuel cell impedance spectra are determined by the cathode. The results showed that the formation of the cathode capacitance was achieved after polarisation of about $200 \mathrm{mV}$ from open circuit after which the cathode capacitance reached a value that remained constant for large currents. Wagner [4] proved that it is possible to separate the overall cell impedance into impedance contributions from the electrode and the electrolytic resistance by simulating the measured EIS with an equivalent circuit. The results showed that the electrode impedances can be separated to represent the impedance of different reaction steps. The use of the EIS technique and the equivalent circuit method have also been used in the work of Jiang et al. [5]. This study compared impedance spectra at various carbon monoxide concentrations mixed with hydrogen. The results showed a pseudo-inductive curve in the positive imaginary part of the complex-impedance-plane that accounts for the higher carbon monoxide concentrations in the anode. Andreaus et al. [6] used a simple model (Randles circuit using a finite diffusion (Warburg) impedance) to fit the fuel cell impedance, varying the membrane thickness and the humidification of the reactants in the PEFC. The results demonstrated that the impedance of the anode increased with membrane thickness. This could be attributed to a decrease in the net flux of water from cathode towards the anode. Wagner [7], included an inductive element in the electrode circuit to represent a relaxation impedance. In other studies [8,9] although the impact of such element during fitting is small the literature suggests that these inductive elements can be related to the flooding of the electrodes. Makharia et al. [10] and Guangchun and Pickup [11] applied the transmission line circuit model to interpret EIS data for cathodes with two different Nafion-to-Carbon (N/C) weight ratios. The results demonstrated that the resistance of the catalyst layer electrolytic network increased with decreasing N/C concentration in the electrode. Lefebvre et al. $[9,12,13,14]$ demonstrated that EIS can be applied to extract detailed information about ion transport in the catalyst layer. Data for catalyst layers with and without impregnated ionomer were compared. The Nafion-containing electrode was shown to have a much higher ionic conductivity and, consequently, had a larger active area and provided better fuel cell performance. Cano-Castillo et al. [9] analysed general flooding characteristics of the PEFC cathode using EIS measurements and the transmission line circuit model. As expected, the proton resistance in the anode catalyst layer decreased by the back-diffusion of water from the cathode to anode, driven by a gradient in its concentration across the separating PEM. As the cathode is the source of water, it is not expected to have high proton resistance but can be subject to flooding, thereby inducing a mass transport effect. Suzuki et al. [14] demonstrated diagnostics techniques to evaluate proton conductivity and gas diffusivity in the catalyst layer. The proton conductivity measurements using the AC impedance method showed a notable dependence on Nafion loading. The gas diffusivity measurement using helium gain revealed the contribution of Knudsen diffusion in the catalyst layer. From these measurements, gas diffusion in the catalyst layer appears to limit the cell performance. Eikerling and Kornyshev [15] constructed impedance models for polymer electrolyte membrane fuel cells and derived analytical solutions for several cases. In this study, the effects of reaction kinetic, double layer capacitance and proton conductivity in the catalyst layer were studied. The results distinguished the contribution of the catalyst layer from the contribution of other fuel cell components and characterised it quantitatively. Devan et al. [16] presented a macrohomogeneous model for a porous electrode that included coupled potential and concentration gradients with linear kinetics. The results showed that concentration gradients become significant at very high exchange current densities. 
As the above review suggests, the frequency response of a PEFC obtained from EIS reflects the response of the entire cell, including the internal layers and the phenomenological processes occurring within them. When an equivalent circuit is fitted to the frequency response of the entire PEFC, it is possible to selectively subtract some of the electrical components from that circuit in order to obtain the transmission line circuit which corresponds solely to one specific catalyst layer. The frequency response of this catalyst layer alone can then be reproduced using the obtained transmission line circuit and previously-measured electrochemical properties of the catalyst layer. In this simulated frequency response, a $45^{\circ}$ straight-line appears at the high frequency end of the spectra. When, for example, a parallel Resistor-Capacitor (RC) circuit is used instead of the transmission line circuit, this straight-line does not appear. The literature demonstrates that this linear region can be associated to the proton transport in the catalyst layer $[10,12]$ and that its value can be calculated from the projection of this section onto the real axis.

The objective of the current research is to generate a numerical model to predict the impedance spectrum of the CCL at low currents based on fundamental electrode theory and measurable properties of the CCL, and to apply the model to determine characteristic transient and spatial profiles for the layer. By coupling the fundamental theory of fuel cell processes with measurement-based impedance analysis, it is possible to open a wider scope to generate a greater understanding of how the structure, material composition and operating conditions of the PEFC govern cell performance. The current study begins by stating the electrochemical reaction rates on a thickness basis to define the rate of current generation across the CCL. Capacitance effects and proton resistance are subsequently considered in the expression of current. The theoretical treatment in the time domain due to the charge capacitance is then translated into the frequency domain which leads towards a mathematical expression to simulate the impedance spectrum of the CCL. The physics of the catalyst layer (double layer capacitance, charge transfer resistance across the electrode-electrolyte interface and proton resistance in the electrolytic network) are obtained from a softwarebased process where the experimental impedance curves for a PEFC are fitted to the equivalent circuit of the PEFC. The simulated frequency response from the numerical model is then validated with experimental curves at three operating temperatures. The final part of the study considers an application of the validated model in the time domain in order to reveal the transitory and steady-state response of the current as a function of the thickness of the CCL.

\section{Electrochemical Mechanisms of CCL in Time Domain}

The theoretical treatment of the electrochemistry of the CCL in this study is considered in six steps. The first step focuses on the fundamentals of reaction kinetics for the CCL and the definition of the forward and backward rate constants for the oxidation and reduction reactions. This is then modified to incorporate the dependence of reaction rates on temperature. The third steps establishes the basic expression for total current in the catalyst layer. This is subsequently modified to include capacitance effects in the CCL, and the resulting equation is then linearised. The fifth step introduces proton resistance, which leads to an expression in the time domain that can be solved to determine spatial and temporal current distributions in the CCL. Finally, the key expression in the time domain is translated into the frequency domain to predict the frequency response of the CCL. 
The main assumptions and considerations for the current theoretical treatment of the CCL are as follow:

- the mathematical analysis is considered for a PEFC under isobaric and isothermal conditions;

- only effects in the CCL are considered;

- oxygen diffusion limitations in the CCL are negligible at low currents;

- spatial gradients are only considered through the thickness of the CCL, and therefore the model is mathematically one-dimensional;

- the resistance to proton transfer in the electrolyte of the catalyst layer is much greater than the resistance to electron transfer in the carbon of the catalyst layer by several orders of magnitude [10]. Therefore electronic Ohmic loss in CCL can be regarded as being negligible. The literature suggests that this is a reasonable assumption when using a high-frequency resistance meter to estimate the resistivity of the catalyst layer [14];

- the metal alloy deposits supported on carbon and the dispersion of polymer electrolyte are spread homogenously in the CCL;

\subsection{The current in the electrochemical reaction}

The electrochemical reaction occurring at the electrode takes the form of :

$$
\mathrm{Ox}+e^{-} \stackrel{k}{\longleftrightarrow} \operatorname{Re}
$$

where Ox is the oxidized form of the chemical species and $R e$ is the reduced form of the chemical species. If the potential of the electrode is made more negative than the equilibrium potential (reversible potential), the reaction will form more Re. If the potential of the electrode is more positive than the equilibrium potential, it will create more Ox. The chemical reaction proceeds at different rates. The factors that affect reaction rates include the surface area of a catalyst site, temperature, the phase of the reactants (i.e., gas or liquid) and the catalytic loading. A change in one or more of these factors may alter the rate of a reaction. When a catalyst is used (platinum in a fuel cell) the reaction rate can be stated on a catalyst weight or surface area basis. In this analysis thickness basis was considered.

For the reaction (1) the flux of the chemical species through the thickness of the catalyst layer is given by:

$$
\frac{d v_{i}}{d x}=k_{i} c_{s}
$$

where the subscript $i$ denotes the forward and the backward reactions, and $s$ denotes the reduced specie and the oxidized specie; $v$ is the flux of the species, $k$ is the reaction rate coefficient and $c$ is the surface concentration of the chemical species respectively. Substituting the Faraday's law into the flux of the species yields an expression for the forward and backward current $j$ :

$\frac{d j_{i}}{d x}=z F k_{i} c_{s}$

where $z$ is the number of electrons released or consumed, and $F$ is the Faraday constant.

Another factor altering the rate of the reaction is the temperature. This can be expressed using the Arrhenius equation [17]. If the potential in equilibrium of the chemical reaction is changed to a new value, the Gibbs free energy will change as well. The forward and backward reaction rate coefficient are: 
$k_{i}=A k^{0} \exp \left(\frac{\alpha_{i} z F\left(E^{o}-E\right)}{R T}\right)$

where $A$ is the electrode area, $k^{0}{ }^{1}$ is called the standard rate constant, $R$ is the ideal gas constant, $E^{o}$ is the potential in equilibrium, $E$ is the local potential across the catalyst layer away from equilibrium and $T$ is the temperature. For the forward reaction, it is $\alpha_{i}=\alpha$, and for the backward reaction, it is $\alpha_{i}=-(1-\alpha)$, where $\alpha$ is the charge transfer coefficient. Substituting Eq.(4) into the forward and backward current, Eq. (3), yields:

$\frac{d j_{i}}{d x}=z F c_{s} A k^{0} \exp \left(\frac{\alpha_{i} z F\left(E^{o}-E\right)}{R T}\right)$

Under equilibrium conditions, the reaction (1) proceeds in both directions simultaneously at the same rate. Therefore the potential in equilibrium is equal to the potential of the electrode, $E^{o}=E$. In addition, the concentration (denominated as bulk concentration under this state) are the same for the chemical species, $c_{s}^{*}$. The rate at which the forward and backward transfer of charge occurs at equilibrium is known as the exchange current, $j_{O}$. As such:

$j_{O}=z F c_{s}^{*} A k^{0}$

Under non-equilibrium when an external load is connected to a PEFC a current flows through the electrodes. The total current is the difference between the forward current and the backward current and can be expressed as:

$\frac{d j}{d x}=z F c_{O} A k^{0} \exp \left(\frac{\alpha z F\left(E^{o}-E\right)}{R T}\right)-z F c_{R} A k^{0} \exp \left(\frac{-(1-\alpha) z F\left(E^{o}-E\right)}{R T}\right)$

where the subscript $\mathrm{O}$ denotes oxidation and $\mathrm{R}$ denotes reduction of the chemical species. A relationship between the potential in equilibrium with regard to the non-equilibrium potential can be expressed as $\eta=E_{O}-E$, where $\eta$ is a deviation from both potentials and represents the local over potential across the catalyst layer. Substituting this relationship into the expression of total current Eq.(7), and multiplying both terms by the bulk concentration and substituting the exchange current defined in Eq.(6) gives:

$\frac{d j}{d x}=\frac{c_{O}}{c_{O}^{*}} j_{O} \exp \left(\frac{\alpha z F \eta}{R T}\right)-\frac{c_{R}}{c_{R}^{*}} j_{O} \exp \left(\frac{-(1-\alpha) z F \eta}{R T}\right)$

At low currents there are limited mass transportation effects therefore it is considered that there is no effect on the concentration distribution through the CCL. It can be assumed that $c_{s}=c_{s}^{*}$ for both reduction and oxidation processes. In addition, when the overpotential increases the contribution of the second term of the right side in Eq.(8) becomes small and therefore it can be neglected. The final equation is the rate of current generation across the catalyst layer is

$\frac{d j}{d x}=j_{O} \exp \left(\frac{\alpha z F \eta}{R T}\right)$

\footnotetext{
${ }^{1}$ In the electrochemical literature sometimes it is also called the intrinsic rate constant
} 
The catalyst layer is formed by a double layer structure for the interface between the dissimilar materials, i.e., the electrode-electrolyte interface. At this interface an electric field is presented and has a determinant role in the charge distribution within the reactants as well as in the position and orientation of the reactant to form the desired products. This double layer can behave like a capacitor $C$ that is in parallel with the electrode reactions. As such, the current passing from the electrode to the electrolyte either can take part in the charge transfer reactions or can contribute to the charge in the capacitive effect,

$$
\frac{d j}{d x}=j_{0} \exp \left(\frac{\alpha z F \eta}{R T}\right)+C \frac{d \eta}{d t}
$$

Integrating and rearranging Eq.(10) yields;

$$
\frac{d \eta}{d t}=\frac{1}{C} \frac{j}{x}-\frac{j_{0}}{C} \exp \left(\frac{\eta}{b}\right)
$$

where $b=R T /[\alpha z F]$. Eq.(11) is inherently nonlinear and considers an input variable $j$, and an output variable $\eta$; however it is necessary to approximate the response of this nonlinear system to that represented by a linear system around one point $\eta_{s}$ which represents the steady state of the nonlinear system.

Linearity in electrochemical systems is controlled by potential. The use of a low-amplitude voltage in the EIS technique allows the application of a linear model in order to interpret the impedance spectra. For systems presenting a linear current-voltage relationship, a very large amplitude can be used. For systems presenting a nonlinear current-voltage relationship, a much smaller amplitude is needed.

An electrochemical system that follows the Tafel law is polarized at a potential $\eta_{S}$ on the polarization curve. If a potential is superimposed by EIS, the current response can be calculated using the Taylor series expansion. As such, a linear equation to represent the current after superimposing a potential can be obtained by applying the mathematical treatment described in Appendix A-1 to Eq. (11) as:

$$
\frac{d \bar{j}}{d x}=\frac{j_{0}}{b} \exp \left(\frac{\eta_{s}}{b}\right) \bar{\eta}+C \frac{d \bar{\eta}}{d t}
$$

\subsection{Proton Resistance and Current Distribution in CCL}

As discussed, the current passing through the electrode-electrolyte interface presents a capacitive behaviour due to an electrical field and consequently a potential difference between the dissimilar materials. The potential difference is presented as $\bar{\eta}=\eta_{\text {ELECTROLYTE }}-\eta_{\text {ELECTRODE }}$. Substituting into Eq.(12) gives:

$$
\frac{d \bar{j}}{d x}=\frac{j_{0}}{b} \exp \left(\frac{\eta_{S}}{b}\right)\left(\eta_{\text {ELECTROLYTE }}-\eta_{\text {ELECTRODE }}\right)+C \frac{d\left(\eta_{\text {ELECTROLYTE }}-\eta_{\text {ELECTRODE }}\right)}{d t}
$$

Using Ohms law, the potential in the electrolytic network can be defined as:

$$
\frac{d \eta_{\text {ELECTROLYTE }}}{d x}=R_{P} \bar{j}
$$

where $R_{p}$ is the resistance to the flow of protons in the electrolyte network. It is assumed that the resistance to electron flow in the electrode network is smaller than the proton resistance in the electrolytic network [10] and so 
the electrode network can be neglected $\bar{\eta}=\eta_{\text {ELECTROLYTE }}$. Substituting Eq.(13) into Eq.(14), the electrochemical mechanisms of the CCL, gives the total current distribution in the CCL in time domain,

$\frac{d^{2} \bar{j}}{d x^{2}}=\frac{R_{P} j_{0}}{b} \exp \left(\frac{\eta_{S}}{b}\right) \bar{j}+R_{P} C \frac{d \bar{j}}{d t}$

which is a second-order partial differential equation and can be solved in one dimension using the pdepe command in Matlab [18].

\section{The Electrochemical Mechanisms of CCL in the Frequency Domain}

The electrochemical reaction in a PEFC results in an inhomogeneous distribution of charge in the catalyst layer [19]. As a result, a non-ideal capacitive behaviour has to be considered in the theoretical treatment. In order to correct for this inhomogeneity, a Constant Phase Element (CPE) $Y(i \omega)^{P}$ is used in Eq.(15) to replace the capacitor in the electrochemical mechanisms of the CCL;

$\frac{d^{2} \bar{j}}{d x^{2}}=\frac{R_{P} j_{0}}{b} \exp \left(\frac{\eta_{S}}{b}\right) \bar{j}+R_{P} Y(i \omega)^{P} \bar{j}$

where $Y$ represents a parameter related to CPE and superscript $P$ represents a parameter to correct the inhomogeneity in the distribution of charge. Eq.(16) gives the distribution of current in the catalyst layer in the frequency domain and can take the form of a homogeneous equation which can be solved through the method of $n^{\text {th }}$ order homogeneous equations with constant coefficients, as described in the appendix A-2, which gives:

$\bar{j}=A \exp (\lambda x)+B \exp (-\lambda x)$

In At the GDL-catalyst layer interface, the boundary conditions are $x=0$ and $\bar{j}=0$. At the PEM-catalyst layer interface, the boundary conditions are $x=L$ and $\bar{j}=j_{m}$. $A$ and $B$ are obtained as,

$$
A=-\frac{j_{m}}{\exp (-\lambda L)-\exp (\lambda L)} ; B=\frac{j_{m}}{\exp (-\lambda L)-\exp (\lambda L)}
$$

Substitute $A$ and $B$ into Eq.(17) with trigonometric identities yields the current distribution in the frequency domain:

$\bar{j}=j_{m} \frac{\sinh (\lambda x)}{\sinh (\lambda L)}$

The potential in the frequency domain can be obtained by rearranging Eq.(12) and replace the capacitor by the $\mathrm{CPE}$ in the frequency domain as:

$$
\bar{\eta}=\frac{b}{\exp \left(\frac{\eta_{S}}{b}\right) j_{O}+b Y(i \omega)^{p}} \frac{d \bar{j}}{d x}
$$

where the current differentiation can be obtained by substitute $A$ and $B$ into Eq.(17)

$$
\frac{d \bar{j}}{d x}=j_{m} \lambda \frac{\exp (\lambda x)+\exp (-\lambda x)}{\exp (\lambda L)-\exp (-\lambda L)}
$$

Hence, substituting Eq.(20) into Eq.(19) with trigonometric identities gives the potential in the frequency domain: 
$\bar{\eta}=\frac{\lambda b j_{m} \cosh (\lambda x)}{\left(\exp \left(\frac{\eta_{S}}{b}\right) j_{O}+b Y(i \omega)^{P}\right) \sinh (\lambda L)}$

The impedance of the CCL is defined as the ratio between the potential and current at frequency domain,

$Z=\frac{\bar{\eta}}{\bar{j}}=\frac{\lambda b \operatorname{coth}(\lambda x)}{\exp \left(\frac{\eta_{S}}{b}\right) j_{O}+b Y(i \omega)^{P}}$

The solution to Eq. (22) over a range of frequencies will contain a real and imaginary component, which can be presented on a Nyquist plot.

\section{Impedance Model and Validation}

\subsection{The Impedance Model in the Frequency Domain}

The overall experimental and modelling framework developed for the current study is illustrated in Fig.1. There are in essence two elements of the experimental work; the first element provides the frequency response of a working cell for validation purposes while the second element provides the requisite kinetic parameters of the catalyst layers from electrical test to supplement the theoretical treatment. The modelling work involves two sequential stages; initially, a mechanistic description in the time domain followed by a translation into the frequency domain. Once validated in the frequency domain, results are obtained from the time domain in order to deduce spatial and temporal current gradients.

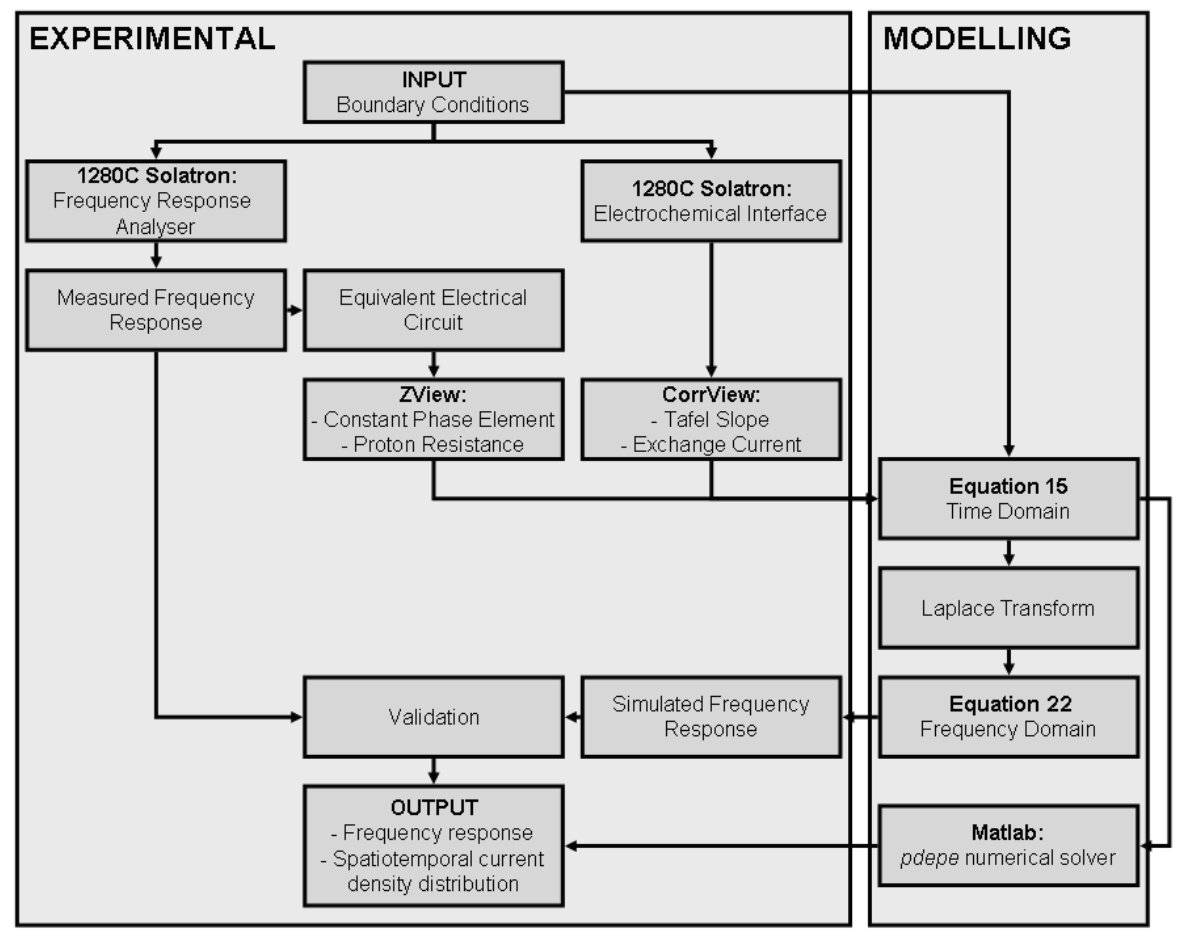

Figure 1: Overall Experimental and Modelling Structure 


\subsection{Experimental Set-up}

A commercial (Electrochem®) $5 \mathrm{~cm}^{2}$ single cell with carbon-supported $0.5 \mathrm{mgPt} / \mathrm{cm}^{2}$ was used for the electrical tests. The cell was supplied with neat hydrogen and oxygen and operated with a dead-ended anode and cathode (stoichiometric ratio of 1) both at a gas pressure of $1 \mathrm{~atm}$. The operating temperature of the cell was adjusted to 30,40 and $60{ }^{\circ} \mathrm{C}$. Both gases were fed dry. EIS measurements were taken at one current of the voltagecurrent curve in the activation zone for the three temperatures with a two-electrode configuration. The tests were performed using a Solartron $1280 \mathrm{C}$ electrochemical interface and a Solartron 1280C frequency response analyzer. The frequency scan was performed from $100 \mathrm{kHz}$ down to $0.1 \mathrm{~Hz}$, with an alternating voltage signal an $10 \mathrm{mv}$ amplitude. To estimate the kinetic parameters, the fuel cell was tested in a three-electrode configuration using CorrWare software.

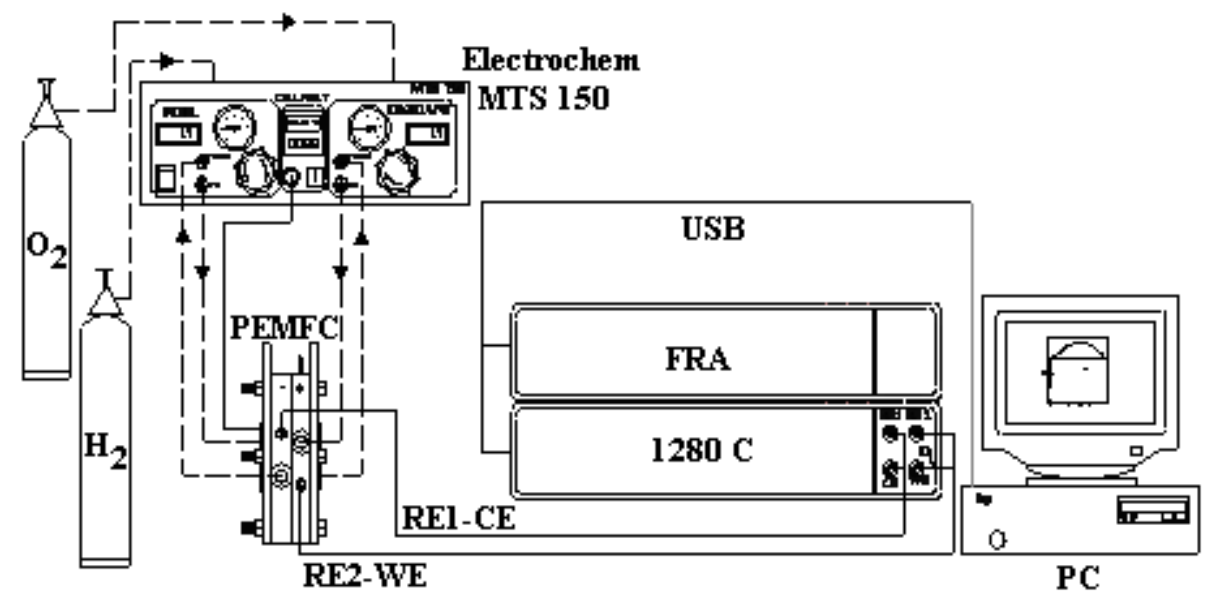

Figure 2. Experimental Setup

\subsection{Measured PEFC Impedance Response}

Figures 3 shows the measured impedance response of the whole PEFC when operating at 30,40 and $60{ }^{\circ} \mathrm{C}$. The results show that the diameter of the impedance spectra reduces with increasing operating temperature. The results also show that for 30 and $40{ }^{\circ} \mathrm{C}$ the high-frequency end of the impedance spectra intercepts the $x$-axis at a higher value than for $60{ }^{\circ} \mathrm{C}$. The vertical segment at the high frequency end reflects the inductance in the electrical cables of the measurement system. This vertical segment does not change in length and therefore demonstrates no significant change with temperature. Therefore, the main differences lie in the arc diameters and the $x$-intercept.

The arc of an impedance spectrum for an entire cell depends upon the kinetics of both electrodes. The reduction in arc diameter with respect to operating temperature occurs because the higher operating temperature fundamentally improves the electrode kinetics. The $\mathrm{x}$-intercept reflects the resistance of the PEM; the lower the value of the $x$-axis intercept, the lower the resistance of the PEM. In the current work, the impedance spectra are 
measured under potentiostatic conditions of $0.8 \mathrm{~V}$. The improved kinetics with temperature allow a greater current to be drawn at this cell potential. At 30,40 and $60^{\circ} \mathrm{C}$, the current drawn is $19,19.6$ and $20 \mathrm{~mA} / \mathrm{cm}^{2}$ respectively. It is possible that the resistance of the cell at $60^{\circ} \mathrm{C}$ could be lower than for the other two temperatures because the amount of water generated within the cell which increases with current density and also due to residual water in the cell as the three tests are conducted sequentially with increasing temperature.

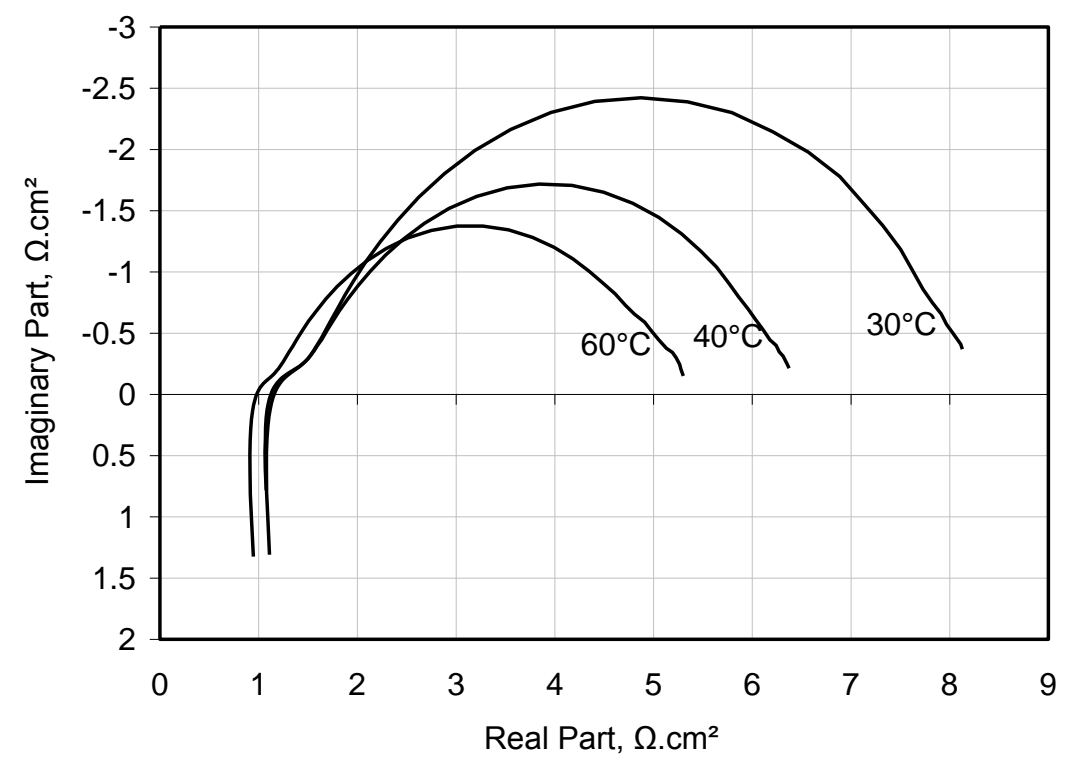

Figure 3. Measured impedance response in a working PEFC at 30,40 and $60^{\circ} \mathrm{C}$.

\subsection{Equivalent Circuit Model}

In order to obtain the parameters of the cathode catalyst layer for the mathematical analysis such as proton resistance and CPE, the EIS was analyzed by adjusting experimental data using a commonly-used equivalent circuit model where each electrode was represented by a transmission line. In this work the Ohmic resistance due to the supporting material in the electrode is neglected. The transmission line of each electrode is connected in series with an inductor element and a resistor. The inductor element accounts for the inductance of the cables used in the test equipment and the Ohmic resistance represents the resistance to proton flow across the PEM. The use of such circuit instead of a mathematical model has been demonstrated in the literature [9].

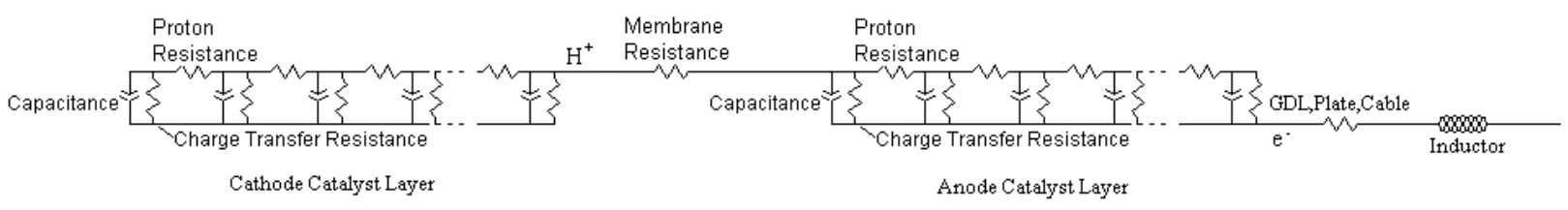

Figure 4. Equivalent circuit model for the PEFC 
As discussed in the literature review, once an equivalent circuit model is fitted to the measured impedance spectrum of the entire cell, the transmission line of the anode catalyst layer, the Ohmic resistor for the PEM and the inductor element are all removed to leave the transmission line of the cathode catalyst layer. This can then be used to generate the AC impedance response of just the cathode catalyst layer in isolation, which is otherwise masked in the measured impedance spectrum of the entire cell.

\subsection{Validation}

In order to validate the theoretical treatment developed in this work, it is necessary to determine the kinetic parameters of the CCL, the proton resistance of the CCL and the capacitive effect of the CCL for Eq. (22). The kinetic parameters are measured using the three-electrode configuration and CorrView software. The proton resistance and the capacitive elements are generated by the ZView software based on the measured impedance spectrum and the transmission line accounting for the CCL as part of the entire equivalent circuit of the PEFC. Table 1 shows the parameters estimated by CorrView and ZView for the current configuration and test cases.

\begin{tabular}{|c|c|c|c|c|c|c|}
\hline \multirow[t]{2}{*}{$\mathrm{T}^{\circ} \mathrm{C}$} & $\mathrm{Rp}$ & Jo & $\mathrm{b}$ & $\mathrm{Y}$ & \multirow[t]{2}{*}{$\mathrm{P}$} & \multirow{2}{*}{$\begin{array}{c}\eta_{S} \\
V\end{array}$} \\
\hline & $\mathrm{m} \Omega . c m^{2}$ & $\times 10^{-3} \mathrm{~A} / \mathrm{cm}^{2}$ & $\times 10^{-3} \mathrm{mV}$ & $\times 10^{-3} \quad S^{P} / \Omega . \mathrm{cm}^{2}$ & & \\
\hline 30 & 900 & 0.065 & 66.88 & 12 & 0.806 & 0.34 \\
\hline 40 & 880 & 0.069 & 62.45 & 12.5 & 0.775 & 0.33 \\
\hline 60 & 600 & 0.073 & 59.28 & 11.4 & 0.787 & 0.32 \\
\hline
\end{tabular}

Table 1. Kinetic, Ohmic and Capacitive parameters of the PEFC estimated by Zview and Corrview software.

The simulated impedance response based on the theoretical treatment is validated against the isolated impedance response of the CCL which is regenerated from the transmission line model of the CCL, as described in section of Equivalent Circuit Model. Fig. 5 shows the comparison between simulated and measured data at 30, 40 and $60^{\circ} \mathrm{C}$. The simulated results were obtained from Eq.(22), and the measured data were from the equivalent electric circuit accounting from CCL. Overall, Fig. 5 shows that with the parameters given in table 1, the theoretical model is capable of simulated the frequency response of the CCL with high accuracy. As with Figs. 3, Fig. 5 demonstrates that the frequency response is highly dependant upon operating temperature due to the effect on electrode kinetics. 


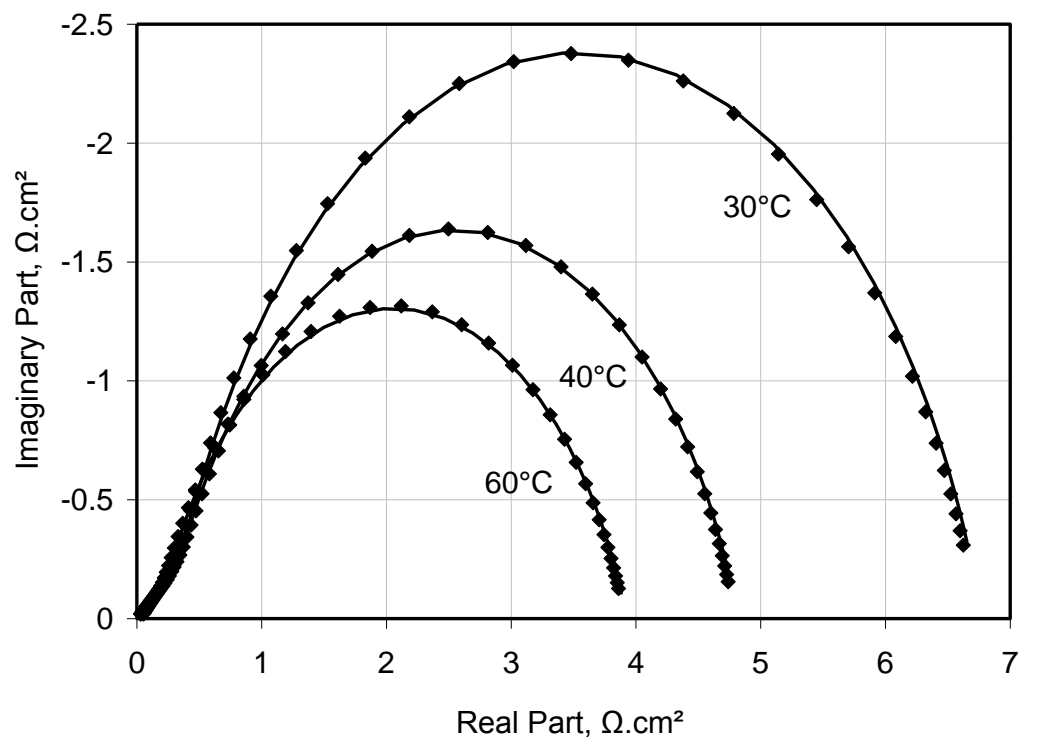

Figure 5. Comparison of impedance between simulated (-) and measured ( $\bullet$ data

\section{Model Application}

One of the main objectives for electrode performance in the PEFC is the optimisation of spatio-temporal current distributions within the catalyst layer. In this section, the effects of proton resistance and the charge capacitance on the current distribution of the CCL are studied. The proton resistance depends on the distribution of electrolyte within the GDL and its hydrated state. The charge capacitance is dependant upon the amount of charge stored between dissimilar materials, i.e., electrode-electrolyte interface.

\subsection{Current Distribution in the Time Domain}

With the validated impedance spectrum of the CCL it is possible to extend the application of the theoretical modelling treatment by using the same parameters as given in Tab. 1 and applying them to the key equation in the time domain, Eq. (15), in order to reveal the spatial and temporal current distributions for the three test cases. This provides a valuable insight into the actual performance of the CCL which results in the impedance spectra given in Figs. 3 and 5.

Figure 6 shows the simulated dynamic response of current along the catalyst layer at $30^{\circ} \mathrm{C}$ and $60^{\circ} \mathrm{C}$. The current distribution increases from the $x=0$ thickness fraction (GDL-CCL interface) to the $x=1$ thickness fraction (CCL-PEM interface). This reflects the transfer of protons from the PEM from the anode catalyst layer, which results in maximum current at the interface between the CCL and the PEM and a minimum current at the interface between the CCL and GDL as protons are consumed due to the oxygen reduction reaction through the thickness of the CCL. The simulations suggest that the current distribution in the CCL reaches steady-state in the order of milliseconds. 


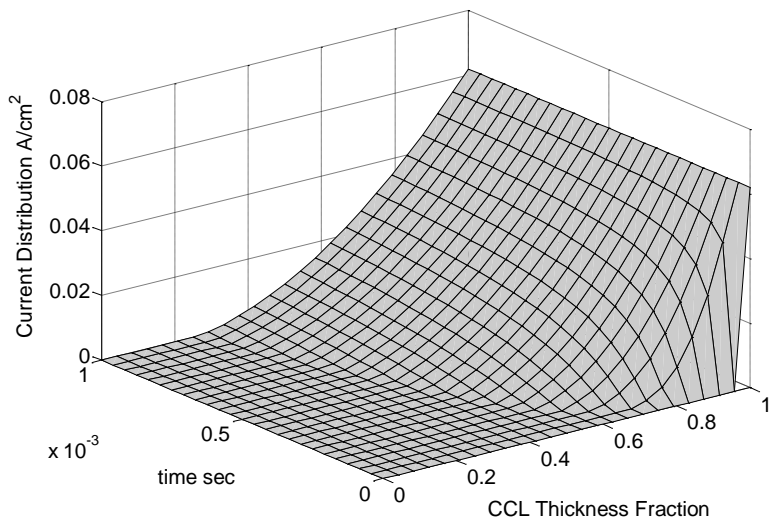

(a)

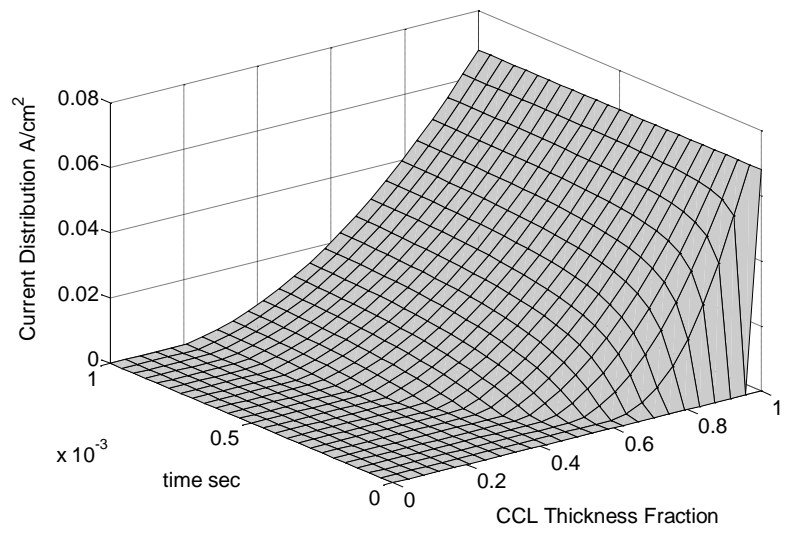

(b)

Figure 6. Simulated dynamic current response along the catalyst layer at (a) $30^{\circ} \mathrm{C}$ and (b) $60{ }^{\circ} \mathrm{C}$

The steady-state current distribution along the catalyst layer can be observed more acutely in Fig. 7a for the two temperatures. At $60^{\circ} \mathrm{C}$, the distribution improves marginally between $x=0.5$ and $x=1.0$, yielding a higher local current at $x=1.0$ than for the $30^{\circ} \mathrm{C}$. This demonstrates that the oxygen reduction reaction occurs to a greater degree in the half of the CCL closest to the PEM. Fig. 7b demonstrates the dynamic response of the CCL at a thickness fraction of 0.8. The thickness fraction of 0.8 is chosen because as Fig. 6 suggests it is a position where the dynamics of the CCL are well pronounced but also relatively close to the PEM-CCL boundary where electro-reduction will be correspondingly high. In general, Fig. $7 \mathrm{~b}$ demonstrates that the time to steady-state improves with temperature. At $60^{\circ} \mathrm{C}$, the current reaches $41 \mathrm{~mA} / \mathrm{cm}^{2}$ in $1 \mathrm{~ms}$. At $30^{\circ} \mathrm{C}$, the current reaches $38 \mathrm{~mA} / \mathrm{cm}^{2}$ in around $1.7 \mathrm{~ms}$.

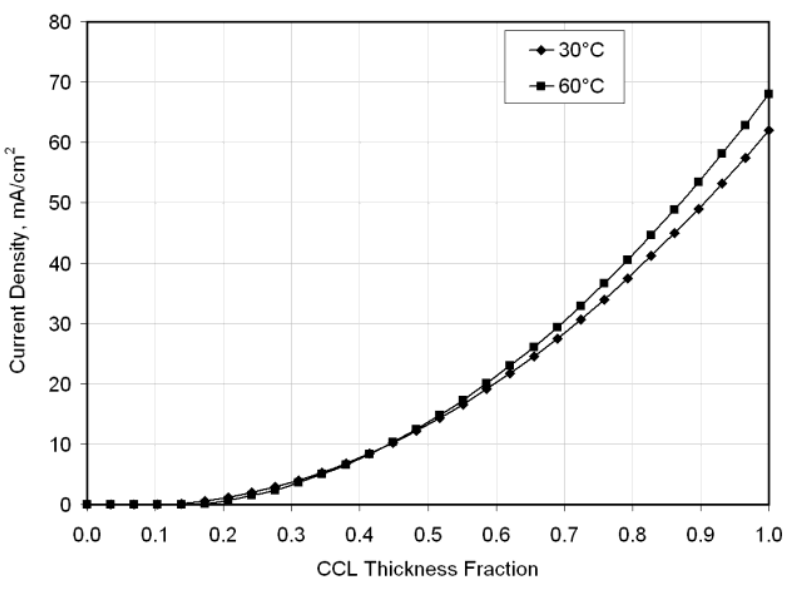

(a)

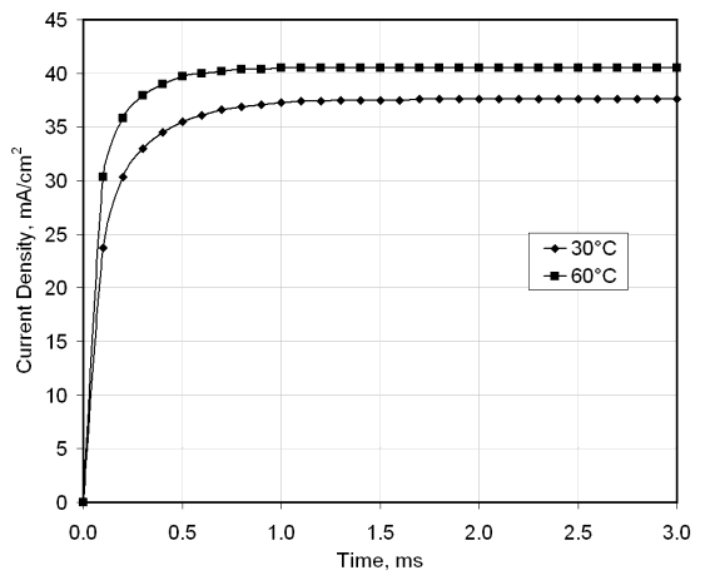

(b)

Figure 7. Current density profiles in the catalyst layer; (a) steady-state current distribution at $t=\infty$; (b) dynamic response of current at a thickness fraction of 0.8 


\subsection{Effect of Double-Layer Capacitance on CCL Performance in the Time Domain}

The purpose of this section is to investigate the effects of double layer capacitance on the performance of the CCL. This is carried out by considering the $60{ }^{\circ} \mathrm{C}$ test case and altering the measured capacitance by $-50 \%,+$ $50 \%$ and $+120 \%$ while all other parameters are kept unchanged.

In this current study, a constant phase element parameter was used to correct the inhomogeneity in the charge distribution of the CCL. This can be observed in the non-perfect semicircle presented in the experimental impedance spectra. The CPE data can be converted to represent a capacitor (measured in Farads) through the following equation:

$C=Y(2 \pi f)^{P-1}$

noting that $f$ is the frequency at which the imaginary part of the impedance reaches its maximum value.

In this parametric study, three simulations are run where the capacitance is altered relative to its measured value in order to investigate the effect of double layer capacitance on current distribution and the dynamic response of the CCL.

Figure 8 demonstrates the effect of the magnitude of the double-layer capacitance on spatial and temporal current distributions in the CCL. From Fig. 8a, it is evident that the capacitance has no significant effect on the distribution of current through the CCL. However, Fig. $8 \mathrm{~b}$ clearly demonstrates that by increasing the double layer capacitance, the time to steady-state is retarded. The steady-state current is approximately $40 \mathrm{~mA} / \mathrm{cm}^{2}$; for the measured baseline capacitance of $5.2 \mathrm{mF} / \mathrm{cm}^{2}$, the model demonstrates that the steady-state current is achieved in the CCL by $1.0 \mathrm{~ms}$. If the capacitance is $50 \%$ less than the measured value $\left(2.6 \mathrm{mF} / \mathrm{cm}^{2}\right)$, the steady-state current is achieved by $0.5 \mathrm{~ms}$. By increasing the capacitance by $50 \%\left(7.8 \mathrm{mF} / \mathrm{cm}^{2}\right)$ and $120 \%\left(11.4 \mathrm{mF} / \mathrm{cm}^{2}\right)$ the time to steady-state is greater and increases to approximately $1.4 \mathrm{~ms}$ and $2.2 \mathrm{~ms}$ respectively.

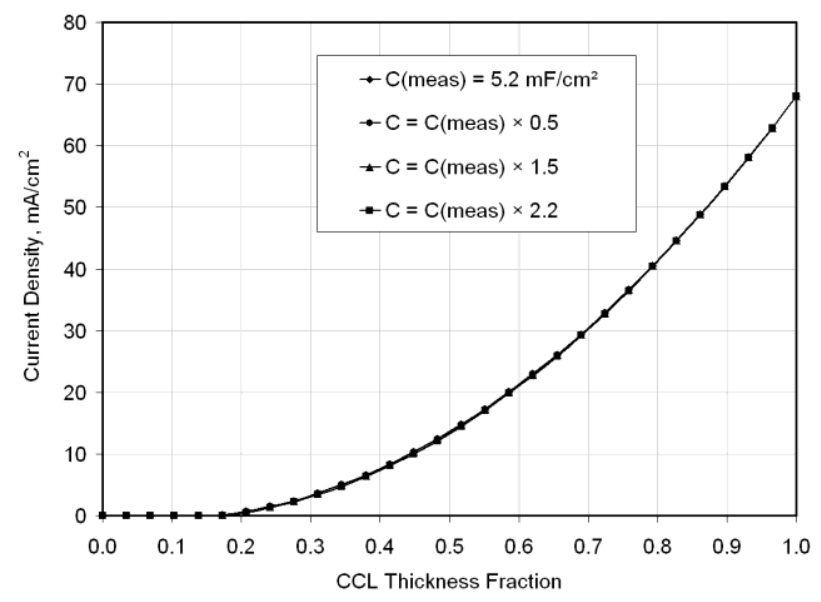

(a)

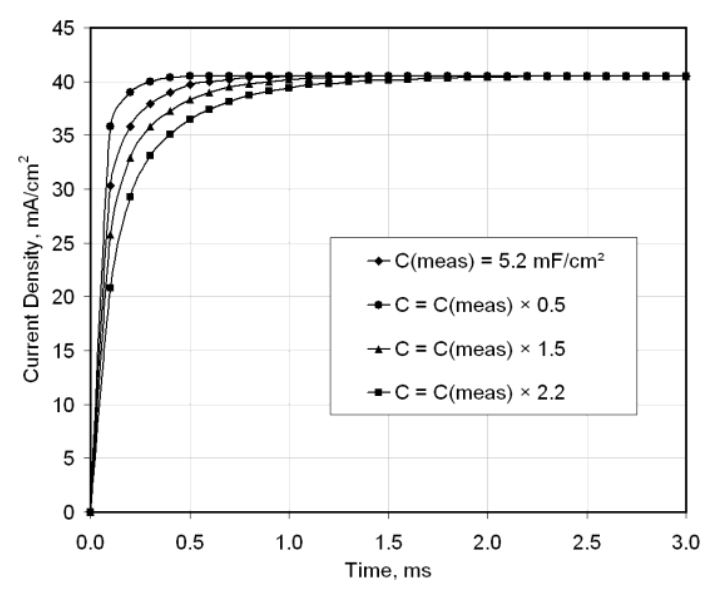

(b)

Figure 8. Effect of double-layer capacitance on CCL current profiles; (a) current distribution at steady-state; (b) dynamic response, $x=0.8$ for different capacitances, $5.2,2.6,7.8$ and $11.4 \mathrm{mF} / \mathrm{cm}^{2}$. 


\subsection{Simulated Effect of Proton Resistance on CCL Performance in time domain}

It is possible to investigate the effect of proton resistance in the CCL on the performance of the CCL in a similar manner to that carried out above. In the current study, two simulations are run where the measured proton resistance $\left(600 \mathrm{~m} \Omega . \mathrm{cm}^{2}\right)$ is decreased by $50 \%\left(300 \mathrm{~m} \Omega . \mathrm{cm}^{2}\right)$ and increased by $50 \%\left(900 \mathrm{~m} \Omega . \mathrm{cm}^{2}\right)$ to once again investigate the effect on current distribution and the dynamic response of the CCL.

Figure 9 demonstrates that the proton resistance has a primary effect on the current distribution and a secondary effect on the time to steady-state. Figure 9a demonstrates that for low proton resistance, the current distribution extends through the thickness of the CCL but can reduce to zero within the CCL if the proton resistance is increased. This suggests that at low current operation when the oxygen distribution within the catalyst layer is assumed to be uniform, high proton resistance can limit the extent of the oxygen reduction reaction to regions closest to the PEM. The high protonic resistance limits the transfer of protons deeper through the electrolyte network towards the CCL-GDL interface, resulting in the curtailed current distribution.

Figure $9 \mathrm{~b}$ demonstrates that while the proton resistance reduces the maximum current achievable at the $\mathrm{x}=$ 0.8 position, the proton resistance also has a secondary effect on the time to steady state. For the three cases considered, the simulated steady state current is approximately $47 \mathrm{~mA} / \mathrm{cm}^{2}, 41 \mathrm{~mA} / \mathrm{cm}^{2}$ and $34 \mathrm{~mA} / \mathrm{cm}^{2}$ for 300 $\mathrm{m} \Omega . \mathrm{cm}^{2}, 600 \mathrm{~m} \Omega . \mathrm{cm}^{2}$ and $900 \mathrm{~m} \Omega . \mathrm{cm}^{2}$ resistances respectively. At $300 \mathrm{~m} \Omega . \mathrm{cm}^{2}$, the steady-state current is achieved in $0.6 \mathrm{~ms}$. At $600 \mathrm{~m} \Omega . \mathrm{cm}^{2}$, the steady-state current is achieved in $1 \mathrm{~ms}$. At $900 \mathrm{~m} \Omega . \mathrm{cm}^{2}$, it takes around 1.3 ms.

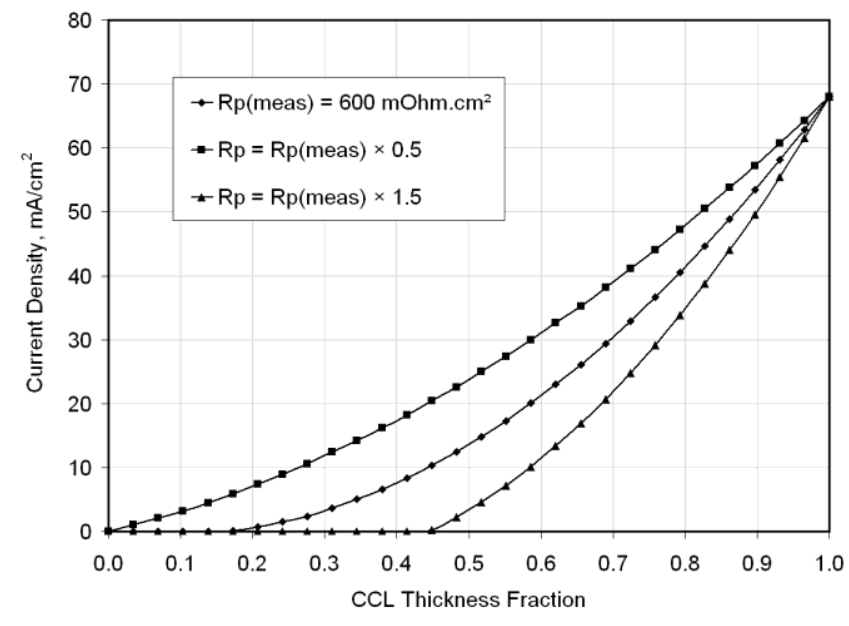

(a)

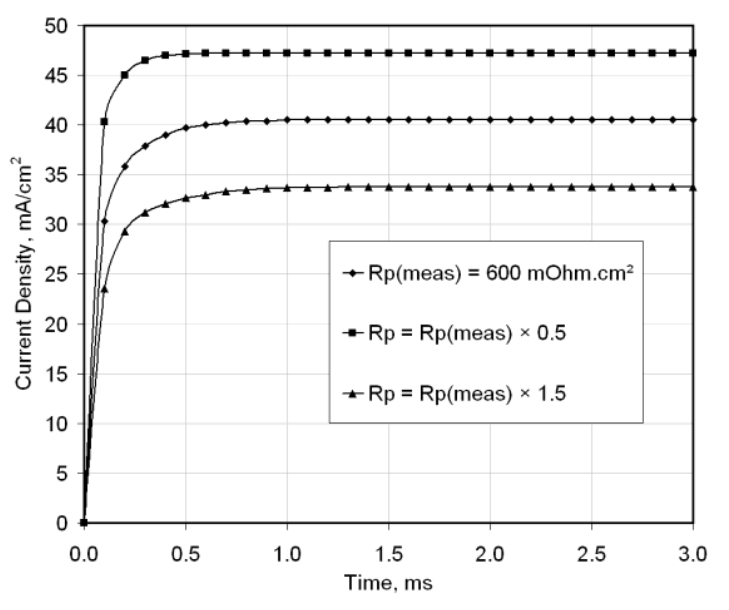

(b)

Figure 9. Effect of proton resistance on CCL current profiles; (a) current distribution at steady-state; (b) dynamic response, $x=0.8$ for different proton resistances, $600,300,900 \mathrm{~m} \Omega . \mathrm{cm}^{2}$ 


\subsection{Discussion}

Fundamentally, double layer capacitance and proton resistance can be related to the electrode-electrolyte networks in the catalytic layer. The capacitance effect is induced by the build-up of charge between the electrode and electrolytic networks. If the interfacial area between the networks increases, the area between which the charge capacitance occurs will increase correspondingly. Therefore, while an increase in the interfacial area may assist in enhancing the utilisation of the full thickness of the catalyst layer, the increase in charge capacitance will have a slight retardation effect on its dynamic response, in the order of milliseconds at low currents (around $20 \mathrm{~mA} / \mathrm{cm}^{2}$ ).

The proton resistance is directly affected by the uptake of water through the electrolytic network. In the current work, while the proton resistance is investigated independently of water transport, the results clearly demonstrate that high protonic resistance in the CCL will limit the current distribution in the CCL and can also have a slight retardation effect on its dynamic response, again in the order of milliseconds. Since high proton resistance inevitably reduces the rate of charge transfer for a given potential by Ohms law, it is evident that it will take longer for the charge to build up between the electrode-electrolyte interface than if the same potential was established with a lower proton resistance and the rate of charge transfer was correspondingly greater. Therefore, the time to steadystate can be improved if the electrical charge is allowed to build up between the interface over a short space of time - for a given potential, this can be achieved if the proton resistance is minimised.

It is arguable that because the time to steady-state at low current is in the order of milliseconds, proton resistance and charge capacitance may have a limited impact on the transient performance of the CCL. In terms of current distribution, however, it is evident from Figs. 8 and 9 that the proton resistance has a significant effect on the utilisation of the catalyst layer and the total current in the CCL at the low current conditions currently simulated. The simulations suggest that by reducing the measured proton resistance by $50 \%$ for the $60^{\circ} \mathrm{C}$ case, the total current in the CCL is increased by $15 \%$ and that current is generated over the full thickness of the CCL as opposed to the $83 \%$ of the CCL closest to the PEM. When the proton resistance is increased by 50\%, the total current in the CCL is reduced by $17 \%$ and current is generated over only $55 \%$ of the thickness of the CCL closest to the PEM.

The results give us an important insight into the balance that has to be achieved between proton resistance and charge capacitance in the CCL, which are both related to the structure and composition of the CCL. At mid current, the spatio-temporal distribution of charge within the CCL may also be affected by mass transport phenomena and therefore the focus of future work will be to extend the current framework to include this effect.

The most important feature of the experimental and theoretical framework that has been developed, verified and applied in this initial study of low current conditions is that it clearly demonstrates the capability to numerically resolve the spatio-temporal distributions of charge within the CCL based on the measured frequency response of the PEFC. 


\section{Conclusions}

The research demonstrates that while EIS serves as an important role in terms of PEFC diagnostics through in-situ impedance spectra and equivalent circuit modelling, a deeper understanding of internal fuel cell processes can be generated by coupling the experimental technique to underlying fundamental theory.

The current study demonstrates that the theory of electrode kinetics can be applied in order to simulate the frequency response of a polymer electrolyte fuel cell cathode catalyst layer at low current densities. The theoretical formulation in the spatial domain is translated into the frequency domain using Laplace transforms. The predicted frequency response of the CCL is validated against that obtained from the measured electrochemical impedance spectra of a $5 \mathrm{~cm}^{2}$ active area PEFC. The frequency response of the CCL is obtained by fitting the measured frequency response of the entire cell to an equivalent electrical circuit model, and reproducing the response of the CCL by extracting a transmission line to represent the CCL in isolation.

The model is validated at three operating temperatures; 30,40 and $60{ }^{\circ} \mathrm{C}$. The results demonstrate excellent agreement between the simulated frequency response of the CCL and that obtained from measurement. The validation therefore confirms the theoretical treatment for low current operation.

The theoretical formulation is solved in the time domain in order to reveal the spatial and temporal distributions of current in the CCL for the three conditions investigated for model validation. The results confirmed that by increasing the operating temperature, it is possible to marginally improve the current distribution through the CCL and the time to steady-state.

As a further extension to this investigation, the effect of double layer capacitance and proton resistance in the CCL were considered separately. With all other parameters unchanged, a decrease in double layer capacitance demonstrated a reduction in the time to steady-state, while a greater capacitance yielded an increase.

The steady-state current distribution was largely unaffected by the double layer capacitance. An increase in the proton resistance resulted in a poorer current distribution across the CCL and a longer time to steady state. It is argued that the double layer capacitance is in part controlled by the total interfacial area between proton- and electron-conducting networks in the CCL. By increasing the interfacial area in the CCL, the double layer capacitance may also increase, thereby prolonging the time to steady-state. By increasing the proton resistance in the $\mathrm{CCL}$, the rate of charge transfer is reduced for a given potential which increases the time it takes for electrical charge to build up between the electrode-electrolyte interface.

\section{Appendix}

\section{A-1 Solution of Linear Equations approximated by the Taylor Series Expansion}

$\frac{d \eta}{d t}=f(\eta, j)$ 
Expanding the second term of Eq. [A-1] which contains the nonlinear term in Taylor series up to its first derivative form, gives

$$
f(\eta, j) \approx f\left(\eta_{S}, j_{S}\right)+\left(\frac{\partial f}{\partial \eta}\right)_{n_{S}, j_{S}}\left(\eta-\eta_{S}\right)+\left(\frac{\partial f}{\partial j}\right)_{\eta_{s}, j_{S}}\left(j-j_{S}\right)
$$

Given that the expansion is carried out around a steady state $\left(\eta_{s}, j_{s}\right)$, Eq. [A-1] can be expressed as

$\frac{d \eta_{s}}{d t}=f\left(\eta_{S}, j_{S}\right)=0$

Since $\eta_{s}$ is a constant, the left side of Eq. [A-1] can be expressed as

$$
\frac{d \eta}{d t}=\frac{d\left(\eta-\eta_{S}\right)}{d t}=\frac{d \bar{\eta}}{d t}
$$

where $\bar{\eta}=\eta-\eta_{S}$ represents the deviation of variable $\eta$ in the steady state $\eta_{s}$. The linear equation can be defined as:

$\frac{d \bar{\eta}}{d t}=\left(\frac{\partial f}{\partial \eta}\right)_{\eta_{s}, j_{s}} \bar{\eta}+\left(\frac{\partial f}{\partial j}\right)_{\eta_{s}, j_{s}} \bar{j}$

\section{A-2 Solution of the Frequency Response by the $\boldsymbol{n}^{\text {th }}$-Order Homogeneous Equations with Constant Coefficients Method}

Equation [16] can take the form of a homogeneous equation:

$a \frac{d^{2} \lambda}{d x^{2}}+b \frac{d \lambda}{d x}+c \lambda=0$

The homogeneous equation can be represented by:

$$
\lambda^{2}+\lambda+\alpha=0
$$

which has the following roots:

$$
\lambda= \pm \sqrt{\left(\frac{R_{P} j_{0}}{b} \exp \left(\frac{\eta_{S}}{b}\right)+R_{P} Y(i \omega)^{P}\right)}
$$


The solution of a homogeneous equation with different roots can be obtained as follows:

$$
\bar{j}(x)=A \exp (\lambda x)+B \exp (-\lambda x)
$$

\section{Nomenclature}

\begin{tabular}{|c|c|c|}
\hline$A$ & active cell area & {$\left[\mathrm{cm}^{2}\right]$} \\
\hline$b$ & tafel slope & {$[m V]$} \\
\hline$c_{O X}, c_{R E}$ & concentration of species & {$\left[\mathrm{mol} / \mathrm{cm}^{3}\right]$} \\
\hline$c_{O X}^{*}, c_{R E}^{*}$ & concentration of species in equilibrium & $\left\lfloor\mathrm{mol} / \mathrm{cm}^{3}\right\rfloor$ \\
\hline$C$ & capacitance between dissimilar materials & {$\left[F / \mathrm{cm}^{2}\right]$} \\
\hline$E$ & potential in electrode far from equilibrium & {$[V]$} \\
\hline$E_{O}$ & potential in electrode in equilibrium & {$[V]$} \\
\hline$F$ & faraday constant & {$[96485 \mathrm{C} / \mathrm{mol}]$} \\
\hline$f$ & frequency & {$[\mathrm{Hz}]$} \\
\hline$G$ & Gibbs free energy of activation & {$[$ joules / mol $]$} \\
\hline$i$ & imaginary component in impedance & \\
\hline$j$ & current density & {$\left[A / \mathrm{cm}^{2}\right]$} \\
\hline$j_{b}, j_{f}$ & backward and forward current & {$\left[A / \mathrm{cm}^{2}\right]$} \\
\hline$j_{o}$ & exchange current density & {$\left[A / \mathrm{cm}^{2}\right]$} \\
\hline$j_{m}$ & maximum rate of proton transfer & $\left\lfloor A / \mathrm{cm}^{2}\right\rfloor$ \\
\hline $\bar{j}$ & current distribution in catalyst layer & $\left\lfloor A / \mathrm{cm}^{2}\right\rfloor$ \\
\hline$k_{b}, k_{f}$ & backward and forward reaction rate coefficient & $\left\lfloor\mathrm{cm} / . \mathrm{s}^{-1}\right\rfloor$ \\
\hline$L$ & thickness of catalyst layer & {$[\mathrm{cm}]$} \\
\hline$\eta$ & relationship between potential far from equilibrium and & \\
\hline & equilibrium potential & {$[V]$} \\
\hline $\bar{\eta}$ & potential distribution in catalyst layer & {$[V]$} \\
\hline$\eta_{S}$ & value of voltage in activation lose where the & \\
\hline
\end{tabular}


impedance is performed

$P \quad$ parameter related to CPE (constant phase element)

$R \quad$ ideal gas constant

$[8.3143 \mathrm{~J} / \mathrm{mol}-\mathrm{K}]$

$R_{P} \quad$ proton resistance in cathode catalyst layer

T temperature

$\left[\Omega . \mathrm{cm}^{2}\right]$

$t$

time

backward and forward reaction flux of the species

angular frequency

$\left\lfloor\mathrm{mol} / \mathrm{cm}^{2} \mathrm{~s}\right\rfloor$

$x$

distance along the catalyst layer

$[\mathrm{rad} / \mathrm{sec}]$

parameter related to CPE (constant phase element)

$[0 \leq x \leq 1]$

$Y$

impedance of cathode catalyst layer

$\left[S^{P} / \Omega . c m^{2}\right]$

$\left[\Omega . \mathrm{cm}^{2}\right]$

$z \quad$ electrons released or consumed

$Z^{\prime} \quad$ real part of impedance

$\left[\Omega . \mathrm{cm}^{2}\right]$

$Z^{\prime \prime} \quad$ imaginary part of impedance

$\left[\Omega . \mathrm{cm}^{2}\right]$

$\alpha$

charge transfer coefficient

\section{References}

${ }^{1}$ Schimidt T.J., Gasteiger H.A., Stab G.D, Urban P.M, Kolb D.M, Behm R.J., Characterization of high surface area electrocatalysts using a rotating disk electrode configuration. J. Electrochemical Soc., 145, 7, (1998), 2354-2358.

${ }^{2}$ Zhang J., PEM Fuel Cell Electrocatalysts and Catalysts Layers, Springer, 2008

${ }^{3}$ Romero T., Arriaga L., Cano-Castillo U., J. Power Sources, 118, (2003)179-182

${ }^{4}$ Wagner N., J. Applied Electrochemistry, 32, (2002), 859-863

5 Jiang R., Kunz R., Fenton J., J. Electrochem. Soc., 152, (2005), A1329-A1340

${ }^{6}$ Andreaus B., McEvoy A., Sherer G., Electrochim. Acta, 47, (2002), 2223-2229

${ }^{7}$ Wagner N., Gulzow E., J. Power Sources., 127, (2004), 341-347.

${ }^{8}$ S.Manzo, U.Cano-Castillo, Study of phenomena in a PEM fuel cell during increasing operating temperature using EIS, Hydrogen Forum HYPOTHESIS VII, Merida, Mexico, March 2007, ISBN: 968-6114-21-1.

${ }^{9}$ U. Cano-Castillo, A. Ortiz, S. Cruz, L. G. Arriaga and G.Orozco, ECS Transactions PEMC 6, V3, Issue I, pp931939, 2006, Editors: T. Fuller et al.

${ }^{10}$ Makharia R., Mathias M.F., Baker D.R., J. of the Electrochemical Soc., 152, 5, (2005), A970-A977

${ }^{11}$ Guangchun L., Pickup P., J. of the Electrochemical Soc., 150, 11, (2003), C745-C752

${ }^{12}$ M.C. Lefebvre, et al., Electrochem. and Solid-State Letters, 2, 6, (1999), 259-261

${ }^{13}$ Junbo H., Wei S., Hongmei Y., Yu Fu, Lixing H. Zhigang S., Baolian Y., J. Power Sources, 176, (2008), 118-121

${ }^{14}$ Suzuki T., Murata H., Hatanaka T., Morimoto Y., Research Report, R\&D Review of Toyota Vol.39 No. 3

${ }^{15}$ Eikerling M. and Kornyshev A.A., J. Electroanalytical Chem., 475, (1999), 107-123.

${ }^{16}$ Devan S., Subramanian V., White R., J. Electrochemical Soc., 151, 6, (2004), A905-A913 
${ }^{17}$ Bard A., Faulkner L., John Wiley, Electrochemical Methods, 2001

18 Matlab, Documentation for Mathworks Products, R2009a, pdepe, Solve initial-boundary value problems for parabolic-elliptic PDEs in 1-D

http://www.mathworks.com/access/helpdesk/help/techdoc/index.html?/access/helpdesk/help/techdoc/ref/pdepe.html

${ }^{19}$ C. H. Hsu, F. Mansfel, Corrosion Science Section, Vol.57, No.9, 2001 